\title{
Restoration of ankle joint, quality of life dynamics and assessment of achilles tendon rupture consequences
}

\author{
Vitomskyi V.V. ${ }^{1,2}$, Lazarieva O.B. ${ }^{1}$, Ra'ad Abdul Hadi Mohammad Alalwan ${ }^{1,3}$, Vitomska M.V. ${ }^{1}$ \\ ${ }^{1}$ National University of Physical Education and Sport of Ukraine, Ukraine \\ ${ }^{2}$ Scientific-Practical Medical Center of Pediatric Cardiology and Cardiac Surgery of the MH of Ukraine, Ukraine \\ ${ }^{3}$ University of Jordan, Jordan
}

\begin{abstract}
Purpose: $\quad$ to investigate the dynamics of restoration of the amplitude of motion in the ankle joint, the quality of life and to assess the effects of the breakdown of the Achilles tendon.

Material: patients ( $n=59$, of which $n=30$ - the main group and $n=29$ - the control group) were examined at 4,8 and 16 weeks after surgery. Indicators registered with the help of: goniometry; the Achilles tendon Total Rupture Score; the scale of an assessment of consequences and results of Leppilahti implications.

Results: $\quad$ the decrease of the total amplitude of the motion in the ankle joint takes place due to the deficiency of the amplitude of the dorsal flexion. At the end of the study the dorsal flexion rates were significantly better among the patients of main group. In particular, its deficit was $3.2 \pm 1.85^{\circ}$ in the main group and $6.8 \pm 2.06^{\circ}$ in the control group. The final total score Me $(25 ; 75)$ was also better according to the questionnaire of the Achilles tendon Total Rupture Score: $82(78 ; 84)$ points against $74(72 ; 77)$ points $(p<0.01)$. An assessment of consequences according to the Leppilahti score was $83.8 \pm 8.58$ points in the main group and $70.7 \pm 10.58$ points in the control group $(p<0.01)$.

Conclusions: means of physical rehabilitation help recover the amplitude of movement in the ankle joint, improve the quality of life and the effects after the rupture of the Achilles tendon. The correct methodological approach and combination of tools further improves the results.

Keywords: physical rehabilitation, therapy, functional abilities, recovery, Achilles tendon, physical activity, physical exercises, quality of life.
\end{abstract}

\section{Introduction}

Injuries and damage of ankle joint are commonly belong to the injuries of the musculoskeletal system. The part of them makes from 6 to $21 \%$ of the total number of traumas of the musculoskeletal system or $40-60 \%$ of the number of the lower limb injuries $[6,15]$. Damage of the tendon-ligament apparatus of the tibia is a major cause to consult a doctor [5]. Subcutaneous rupture of Achilles tendons occupy a leading position and make up $47 \%$ of tendons and muscles rupture $[7,8]$.

The rupture of the Achilles tendon is a sudden and severe trauma, which reduces the quality of life. In the biomechanics of the patient's step there is no phase of rupture and shock. It is the result of a violation of the link of transmission of muscle contraction. Also, the rhythm of walking is disturbed, and motor activity is sharply reduced [10]. Hypokinesia and immobilization cause changes in skeletal muscle, lowering the amplitude of motion. They lead to negative morphological and physiological changes [25] and reduce the functional capabilities of the body systems.

The restoration of the normal function of the operated limb is a long process, which includes the adaptation of the ends of the tendon, the normalization of neurotrophic disturbances and the restoration of motor skills $[2,11]$. Available knowledge of the biology of the tendon and regeneration regulation mechanisms are of great importance in surgery and rehabilitation [14].

Physical rehabilitation is an important moment for

(c) Vitomskyi V.V., Lazarieva O.B., Ra'ad Abdul Hadi Mohammad Alalwan, Vitomska M.V., 2017

doi:10.15561/18189172.2017.0608 achieving optimal functional restoration of the entire limb [26]. Optimization of recovery and working capacity become even more important in sport [3]. However, it is noted that until recently attempts to optimize the postoperative regime after surgical treatment of the tendons' rupture were quite empirical. The main reason: from the side of the time characteristics and the gradation of the mode of physical exercise lacked clear conceptual foundations [12].

The biggest difference between protocols of postoperative rehabilitation takes place in the early postoperative period. Such differences are observed in methods of influencing the tendon in the early stages of healing (during the first three-six weeks) [9, 24]. One note that early mobilization is the most common way of managing postoperative tendons $[16,20,23,24]$. It is also preferred in studies: longitudes for immobilization (with the possibility of movement in the volume of $20^{\circ}$ and walking the day after the operation) [13]; early axial loading [18, 19]; early loading of the tendon [23]. Sufficiently detailed protocols for functional management of patients are presented in the works of Nilsson-Helander K. [21] and Nicklas Olsson [22].

Aim of the study is to identify the features of recovery of the functional indexes of the ankle joint, the quality of life and to assess the consequences after physical rehabilitation at the rupture of the Achilles tendon.

\section{Material and methods}

Participants. The materials of the work were obtained during the research on the basis of the State Institution "Institute of Traumatology and Orthopedics of the 
National Academy of Sciences of Ukraine" and the Consultative-Diagnostic Center of Shevchenko District (Kyiv). Patients $(n=59)$ aged 34 to 57 years (including $67.8 \%$ men and $32.2 \%$ women) participated in the study. Patients were referred for surgery with a diagnosis of "Achilles tendon rupture" for years 2014-2016. Patients were divided into the main group $(\mathrm{MG} ; \mathrm{n}=30)$ and the control group ( $\mathrm{CG} ; \mathrm{n}=29)$. The main group was engaged in the developed rehabilitation program, and the control group in the standard.

Organization of research. The developed rehabilitation program include renewed and expanded complexes such as: isometric and ideomotor exercises; exercises in the pool; varieties of walking; special active exercises for restoration of the amplitude of motion; for the restoration of the plantar flexion strength. in the addition to the developed program were include corrective exercises, exercise with fitball, exercise bicycle, platform BAPS, round-bottomed shoes, physiotherapy methods, massage. In the period after the operation, three periods are allocated: immobilization - 4 weeks (duration of the physical therapy from 15 minutes to 30 minutes 2-3 times a day); the period of partial immobilization -4 weeks (up to 40 minutes twice a day); the period of restoration of strength endurance and strengthening of the musculoskeletal system of the shin -8 weeks (up to 45 minutes, 1-2 times a day).

It was used: goniometry of plantar and dysplastic phlegm of the ankle joint (4, 8 and 16 weeks after surgery); assessment of quality of life according to Achilles tendon Total Rupture Score ( 8 and 16 weeks after surgery). It was conducted the evaluation of the results of patients' treatment according to Leppilahti scale: consists of seven points, six of which have a maximum score of 15; one point is maximally estimated at 10 points [17] (16 weeks after the operation).

Statistical analysis. Materials of the study were worked out in the program of statistical analysis - IBM
SPSS 21. Mathematical processing of numerical data was carried out using methods of variation statistics. The analysis of the correspondence of the type of distribution of the quantitative indices of the normal distribution law was checked by the criterion of Shapiro-Wilka (W). For quantitative indices with normal distribution, the average $(\bar{x})$ and the mean square deviation (S) were determined. For quantitative indices with distinctions from normal distribution were additionally determined the median (Me) and the upper and lower quartiles $(25 \%, 75 \%)$. The Wilcoxon criterion (for independent groups) was used to assess the significance of the difference between the baseline and control groups.

\section{Results}

The results of the statistical analysis of the obtained goniometric indices revealed the peculiarities of their dynamics and the existence of significant differences between the groups (Table 1). According to the results of the angle of planter flexion at the time of four weeks after surgery, the differences between the MG and CG were found $(\mathrm{p}<0.01)$ to be significant (Table 1$)$. The average results were $29.1 \pm 1.19^{\circ}$ in the $\mathrm{MG}$ and $28.1 \pm 1.13^{\circ}$ in the CG. The subsequent examinations in eight and sixteen weeks did not reveal significant differences $(p>0.05)$ in terms of the angle of planter flexion (Table 1). The average results in patients of the $\mathrm{MG}$ were $36.5 \pm 2.61^{\circ}$ for eight weeks and $39.6 \pm 4.27^{\circ}$ for sixteen weeks after surgery. In patients of the $\mathrm{CG}$, these results were $35.8 \pm 3.08^{\circ}$ and $39.1 \pm 4.12^{\circ}$ respectively.

So, the dynamics of the deficiency of the amplitude of plantar flexion in the MG was as follow: in four weeks $-11.5 \pm 3.64^{\circ}$; in eight weeks $-4.2 \pm 2.58^{\circ}$; in the sixteen weeks $1,0 \pm 1,60^{\circ}$. Among the control group, the deficit of plantar flexion was: in four weeks $12.3 \pm 3.90^{\circ}$; in eight weeks $-4,5 \pm 2,06^{\circ}$; in the sixteen weeks $1,2 \pm 1,30^{\circ}$.

According to the results of the statistical analysis of the obtained indicators of the angle of the dorsal flexion,

Table 1. Statistical indices of goniometry in the dynamics of restorative treatment, degrees

\begin{tabular}{|c|c|c|c|c|c|}
\hline \multirow[b]{2}{*}{ Indicators } & \multirow[b]{2}{*}{ Group } & \multirow[b]{2}{*}{$\begin{array}{l}\text { Result of healthy } \\
\text { lower limb }\end{array}$} & \multicolumn{3}{|c|}{ Time after surgery } \\
\hline & & & $\begin{array}{l}4 \text { weeks } \\
\text { Me }(25 ; 75)\end{array}$ & 8 weeks & 16 weeks \\
\hline \multirow{3}{*}{$\begin{array}{l}\text { Angle of } \\
\text { plantar flexion }\end{array}$} & MG & $41(39 ; 45)$ & $29(29 ; 30)$ & $37(35 ; 38)$ & $40(37 ; 43)$ \\
\hline & CG & $41(39 ; 43)$ & $28(27 ; 29)$ & $36(35 ; 38)$ & $40(36 ; 41)$ \\
\hline & $\mathrm{p}$ & $>0,05$ & $<0,01$ & $>0,05$ & $>0,05$ \\
\hline \multirow{3}{*}{ Angle of dorsal flexion } & MG & $15(13 ; 18)$ & $-15(-17 ;-15)$ & $6(5 ; 7)$ & $12(10 ; 13)$ \\
\hline & CG & $14(13 ; 18)$ & $-15(-16 ;-15)$ & $4(3 ; 5)$ & $9(7 ; 10)$ \\
\hline & $\mathrm{p}$ & $>0,05$ & $>0,05$ & $<0,01$ & $<0,01$ \\
\hline \multirow{3}{*}{ General amplitude } & MG & $56(52 ; 64)$ & $14(12 ; 15)$ & $43(40 ; 44)$ & $43(48 ; 56)$ \\
\hline & CG & $55(52 ; 60)$ & $12(11 ; 13)$ & $41(38 ; 43)$ & $49(43 ; 52)$ \\
\hline & $\mathrm{p}$ & $>0,05$ & $<0,05$ & $<0,05$ & $<0,05$ \\
\hline
\end{tabular}

Note: $p-$ the level of reliability. 
at the time of four weeks after the operation between the groups was not found any significant differences $(p>0,05)$ (Table 1). The average results of the MG were $15.7 \pm 1.16^{\circ}$. The average results of the CG were $-15.5 \pm 1.05^{\circ}$. In the eight weeks after the surgery the analysis of the results of the index of the dorsal flexion angle revealed a significant difference between the groups $(p<0.01)$. At this stage, the average values were: in the MG and CG $6.0 \pm 1.10^{\circ}$ and $4.1 \pm 1.34^{\circ}$ respectively. A statistically significant difference was maintained at the time of the final survey $(\mathrm{p}<0.01)$. The average values are set at $11,9 \pm 2,15^{\circ}$ in the MG and $8,4 \pm 1,97^{\circ}$ in the CG.

So, the dynamics of the dorsal flexion amplitude deficiency in the MG was as follow: in four weeks $30.8 \pm 2.64^{\circ}$; in eight weeks $-9,2 \pm 2,65^{\circ}$; at sixteen weeks $3.2 \pm 1.85^{\circ}$. Among the control group, the deficit of plantar flexion was as follow: in the four weeks $30.7 \pm 2.80^{\circ}$; in eight weeks $-11,0 \pm 2,81^{\circ}$; in sixteen weeks, $6.8 \pm 2.06^{\circ}$.

According to the results of the overall amplitude index, for all three examinations of the operated limb, it was found a statistically significant difference $(p<0.05)$. In the main group, the overall amplitude was: in the period of four weeks $13.47 \pm 1.98^{\circ}$; in eight weeks $-42,5 \pm 3,12^{\circ}$; at sixteen $-51.6 \pm 5.98^{\circ}$. Among patients of the CG, the following values were recorded: $12.5 \pm 1.62^{\circ}, 39.9 \pm 3.70^{\circ}$ and $47.4 \pm 5.46^{\circ}$ respectively.

In all three surveys the percentage of decrease in the total amplitude of the motion in the ankle (its deficiency) was statistically significantly different between the groups (Table 2). So at the time of the survey in four weeks after the surgery, the deficiency of the general amplitude was: in the MG $75,4 \pm 2,55 \%$; in the CG $-76.9 \pm 2.39 \%$. In eighth week after the surgery, this indicator improved and

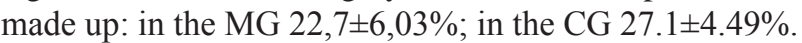
At the final stage, the percentage reduction in the overall amplitude was: in the MG $6.8 \pm 4.28 \%$; in the $\mathrm{CG}-$ $13,7 \pm 3,64 \%$.

It should also be noted that the withdrawal of the ankle joint at $0^{\circ}$ was observed on average: among the patients from the MG - by $11.7 \pm 1.12$ days after the removal of gypsum immobilization [at Me $(25 ; 75)-12(11 ; 13)$ days]; among the patients of the $\mathrm{CG}-13.2 \pm 1.62$ days $(\mathrm{Me}(25 ; 75)-13(12 ; 14))$. The difference between this indicator was statistically significant $(\mathrm{p}<0.01)$

The dynamics of quality of life are the main criterion for the effectiveness of physical rehabilitation [4]. The dynamics of quality of life was studied on the results of the questionnaire (Table 2), which is related to the symptoms and level of physical activity of patients.

The dynamics of Me $(25 ; 75)$ indicators of the total quality of life score have the following features. The first application of the questionnaire in the eight weeks after the surgery has statistically better results in the MG. In the $\mathrm{CG}$, the scores are lower $(\mathrm{p}<0.01)$, the value of Me $(25 ; 75)$ is: in the $\mathrm{MG}-41(38 ; 44)$ points; in the $\mathrm{CG}-$ $36(34 ; 39)$ points. The following and final questionnaires revealed that, at the sixteenth week after the surgery, the results of the general score increased in the groups of patients. The results remained significantly different $(p<0.01)$ with the advantage in the main group (Table 5.6). Indicators of $\mathrm{Me}(25 ; 75)$ after the completion of the course of physical rehabilitation are as follow: in the $\mathrm{MG}$ - $82(78 ; 84)$ points; in the $\mathrm{CG}-74(72 ; 77)$ points.

For evaluation of the results of treatment and consequences it was used the Leppilahti at al. Scale of assessment of the results of patients' treatment [17].

According to the pain in the Scale of assessment both groups have high score (Table 3 ). The statistical difference in pain was not established $(\mathrm{p}>0.05)$. Note that the average score was: in the $\mathrm{MG}-14.3 \pm 1.73$ points; in the $\mathrm{CG}-13,8 \pm 2,18$ points.

The indicator of the MG and CG significantly differed from the point of the scale of "stiffness" $(p<0.01)$. The statistical results were better in the MG (Table 3). The average value is set at the level: in the $\mathrm{MG}-14,7 \pm 1,27$ points; in the $\mathrm{CG}-12.6 \pm 2.54$ points.

The statistical analysis of the subjective characteristic of the force of plantar flexors (the III point of the scale) established the existence of a significant difference between the results of the groups $(p<0,01)$. Thus, the patients of the MG have significantly better results (Table $3)$. In addition, the average value was: in the MG $8.7 \pm 2.92$ points; in the $\mathrm{CG}-6.7 \pm 2.42$ points.

The statistical analysis of observations according to the "restriction in shoes" revealed a significant difference between the groups $(p<0.01)$. This fact confirms the presence of better scores among the patients of the MG (Table 3). Also the higher was an average score of the patients of the $\mathrm{MG}-8.7 \pm 2.25$ points. The $\mathrm{CG}$ had $6.9 \pm 2.47$ points.

The "amplitude" point reflects the degree of difference between the ranges of the ankle joints on the healthy and injured limbs of the patient. The "amplitude" point significantly differed $(\mathrm{p}<0.01)$ in the MG and CG (Table $3)$. Note that the average value was: in the $\mathrm{MG}-13.2 \pm 2.45$ points; in the $C G-10.0 \pm 3.27$ points.

The point "subjective treatment result" indicated satisfaction with the result of treatment by the patient himself. This point had statistically significant difference between the average results of groups of patients $(p<0.01)$. Note that the average score was: in the MG - 14.2 \pm 1.90 points; in the $\mathrm{CG}-12,1 \pm 2,51$ points.

According to the results of the "isokinetic strength" the statistical difference was also better in the MG $(p<0,05)$. The average values of the indicator in the groups of patients were: the $\mathrm{MG}-10,2 \pm 2,78$ points; the $\mathrm{CG}-8,6 \pm 2,27$ points.

The total score by the Scale of assessment [17] was also significantly $(\mathrm{p}<0.01)$ better among the patients who have reducing treatment on the developed program. Note that the average values in the groups were: in the $\mathrm{MG}-$ $83.8 \pm 8.58$; in the $\mathrm{CG}-70,7 \pm 10,58$ points.

In addition, in the distribution of the total score of the scale (according to Leppilahti at al. [17]) were revealed the peculiarities in the gradation (Figure 1): more than 90 points - excellent result; $89-75$ points - good, 74-60 - satisfactory; less than 60 - unsatisfactory. Among the patients of the CG predominate the score as "excellent" 
Table 2. Statistical indicators of quality of life (Achilles tendon total Rupture Score) in the dynamics of rehabilitation, scores

\begin{tabular}{|c|c|c|c|}
\hline Index & Group & $\begin{array}{l}\text { Time after sur } \\
8 \text { weeks } \\
\text { Me }(25 ; 75)\end{array}$ & 16 weeks \\
\hline \multirow{2}{*}{ Total score } & MG & $41(38 ; 44)^{* *}$ & $82(78 ; 84)^{* *}$ \\
\hline & CG & $36(34 ; 39)$ & $74(72 ; 77)$ \\
\hline \multirow{2}{*}{ Limitation at force } & MG & $4(3 ; 4)^{* *}$ & $7(7 ; 8)^{*}$ \\
\hline & CG & $3(2 ; 3)$ & $7(6 ; 8)$ \\
\hline \multirow{2}{*}{ Limitation through tiredness } & MG & $4(4 ; 5)^{*}$ & $8(7 ; 8)^{*}$ \\
\hline & CG & $4(3 ; 4)$ & $7(7 ; 8)$ \\
\hline \multirow{2}{*}{ Limitation through immobility } & MG & $5(4 ; 6)^{* *}$ & $8,5(8 ; 9)^{* *}$ \\
\hline & CG & $4(3 ; 5)$ & $7(6 ; 8)$ \\
\hline \multirow{2}{*}{ Limitation through pain } & MG & $5(4 ; 6)^{*}$ & $8(7 ; 9)^{* *}$ \\
\hline & CG & $4(3 ; 5)$ & $7(7 ; 8)$ \\
\hline \multirow{2}{*}{ Limitation in everyday activity } & MG & $7(6 ; 7)^{* *}$ & $9(8 ; 9)^{* *}$ \\
\hline & CG & $6(4 ; 7)$ & $8(7 ; 8)$ \\
\hline \multirow{2}{*}{ Limitation in walking on uneven surface } & MG & $5(4 ; 6)^{*}$ & $8(8 ; 9)^{* *}$ \\
\hline & CG & $4(3 ; 5)$ & $7(7 ; 8)$ \\
\hline \multirow{2}{*}{ Limitation in fast stair climbing } & MG & $3(3 ; 3)$ & $9(8 ; 9)^{* *}$ \\
\hline & CG & $3(3 ; 3)$ & $8(7 ; 8)$ \\
\hline \multirow{2}{*}{ Limitation in running } & MG & $1(0 ; 2)$ & $8(7 ; 8)^{* *}$ \\
\hline & CG & $1(0 ; 2)$ & $7(7 ; 8)$ \\
\hline \multirow{2}{*}{ Limitation in jumping } & MG & $1(0 ; 2)$ & $8(7 ; 8)^{* *}$ \\
\hline & CG & $1(0 ; 2)$ & $7(7 ; 8)$ \\
\hline \multirow{2}{*}{ Limitation in hard physical activity } & MG & $6(6 ; 7)$ & $8(8 ; 9)$ \\
\hline & CG & $6(6 ; 7)$ & $8(8 ; 9)$ \\
\hline
\end{tabular}

Note. ${ }^{*}$ - the difference between the indicator is statistically significant comparing to the control group at the level $p<0,05 ; * *-p<0.01$.

Table 3. Statistical measures by the Scale of assessment (by Leppilahti at al. [17]) after rehabilitation, scores

\begin{tabular}{lll}
\hline Index & $\begin{array}{l}\text { Group } \\
\text { MG } \\
\text { Me (25\%; 75\%) }\end{array}$ & CG \\
\hline Pain & $15(15 ; 15)$ & $15(15 ; 15)$ \\
Stiffness & $15(15 ; 15)^{* *}$ & $15(10 ; 15)$ \\
The strength of plantar flexors (subjectively) & $10(5 ; 10)^{* *}$ & $5(5 ; 10)$ \\
The restriction in shoes & $10(5 ; 10)^{* *}$ & $5(5 ; 10)$ \\
Amplitude & $15(10 ; 15)^{* *}$ & $10(10 ; 10)$ \\
Subjective result & $15(15 ; 15)^{* *}$ & $10(10 ; 15)$ \\
Isokinetic strength & $10(10 ; 10)^{*}$ & $10(5 ; 10)$ \\
Total score & $85(80 ; 90)^{* *}$ & $70(65 ; 80)$ \\
\hline
\end{tabular}

Note. * - the difference between the indicator is statistically significant compared to the control group at the level $p<0,05 ; * *-p<0.01$. 


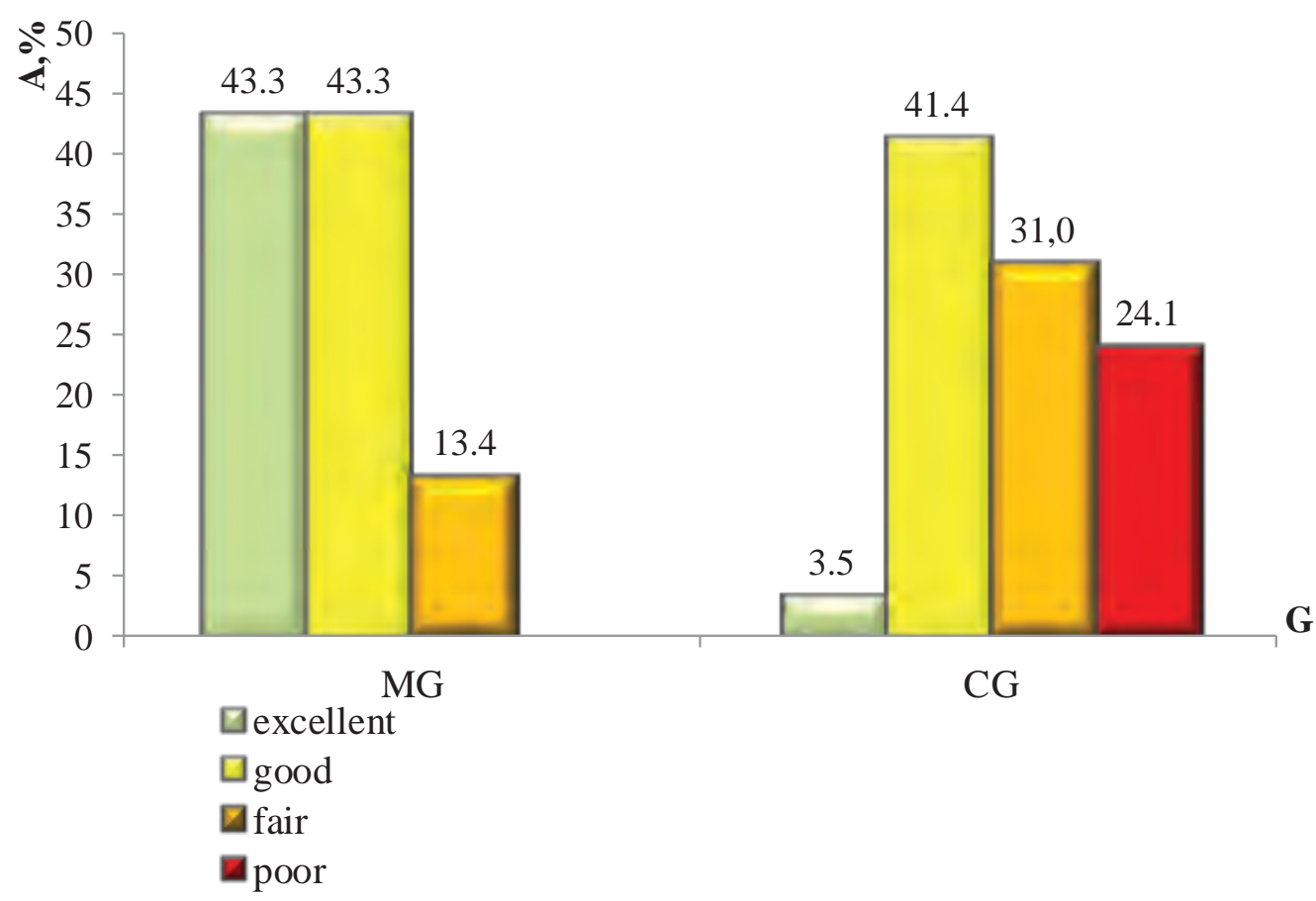

Fig. 1. Distribution of the total score of the scale for the evaluation of the results of patients' treatment with Achilles tendon ruptures (according to Leppilahti at al. [17]) according to the gradation of the results: A - percentage of patients,\%; G group; MG - main group; CG - control group; excellent - excellent result (excellent); good - good result (good); fair - satisfactory result (fair); poor - unsatisfactory result (poor).

and "good" (by 43.3\%). Other patients (13.4\%) had satisfactory treatment results. Among the patients of the $\mathrm{CG}$, the distribution of indicators was worse. In the CG only a small percentage of patients $(3.5 \%)$ were rated "excellent". The largest proportion of patients in the CG was rated "good" (41.4\%).

An essential proportion (31\%) of patients of the CG received satisfactory result. In the $\mathrm{CG}$, an unsatisfactory result was $24.1 \%$. There was no satisfactory result in the MG.

\section{Discussion}

The obtained results point to the possibility of improving the effectiveness of physical rehabilitation in the application of a more intense program. However, the algorithm for rehabilitation should never be transformed into a template. When creating a rehabilitation program, one should give preference to a method that can restore the performance of the patient with the lowest risk and in the shortest time with the best anatomical and functional result.

The dynamics of the indicators shows that the level of restoration of the functional state of the joint and modern methodological approaches to the construction of the rehabilitation program affect the quality of life of the patient.

The conducted research and the obtained results confirm statistical data on the dynamics of goniometric indices during restorative treatment. Thus, in the work of Ayub Hussein Mussa [1], it was reported that the athlete group did not differ from the control group of athletes after the surgical treatment of the Achilles tendon and the course of accelerated and intensive rehabilitation (the standard rehabilitation program was used). But according to the index of dorsal flexion there were significant differences between the groups of athletes. Thus, in this study the groups of athletes had similar dynamics in goniometry indices.

The research of Lantto I. [16] concerned the evaluation of long-term results $(11.0 \pm 0.9$ years $)$ of the usage of various protocols of monitoring patients in the early postoperative period. The author found that the average score on Leppilahti scale was: in the group with early mobilization and use of the brace $-92.9 \pm 5.6$; in the group with immobilization in a stretched state $-93,6 \pm 7,2$ points. No statistical differences were found.

Willits K. et al. [27] investigated patients who had surgical intervention and conservative treatment. In two years after the acute rupture of Achilles tendon according to Leppilahti scale the indicators were as follow: 82 points - among patients who had surgical intervention; 83 points - among patients in the group of conservative treatment. These values are considered good, but not different.

Thus, the study of the effectiveness of surgical treatment, and rehabilitation measures should take into account biomechanical parameters and quality of life of patients.

\section{Conclusions}

The rupture of the Achilles tendon and immobilization after surgical treatment lead to a decrease in the amplitude of the dorsal and plantar flexion, quality of life. 
A condition for improving the dynamics and results of the recovery process in patients after the Achilles tendon ruptures is: modern methodological approaches in the organization of the rehabilitation process; taking into account the philosophy of the international classification of functioning and methods of determining the SMART goals; appropriate combination of means.

The restoration of the amplitude of the dorsal flexion is more slowly than planar. The dynamics of restoration of functional indicators of the lower limb and quality of life was significantly better among patients undergoing rehabilitation course on the developed program.

\section{Acknowledgments}

The research is carried out in accordance with: "The consolidated plan of research work in the field of physical culture and sports for years 2011-2015" on the topic 4.4. "The improvement of organizational and methodological bases of programming of the physical rehabilitation process during dysfunctional disorders in various systems of the human body" (state registration number 0111U001737); "Plan of research work of the National University of Physical Education and Sports of Ukraine for years 20162020" on theme 4.2. "Organizational and theoretical and methodological bases of physical rehabilitation of persons of different nosological, professional and age groups" (state registration number 0116U001609).

\section{Conflict of interest}

The authors state that there is no conflict of interest.

\section{5}

13.Cetti R. Ruptured achilles tendon - preliminary results of a new treatment. Br. J. Sports Med. 1988; 22(1):6-8.

14.Evans NA, Stanish WD. The basic science of tendon injuries. Current Orthopaedics. 2000;14(6):403-412.

15.Henari S, Banks LN, Radovanovic I, Queally J, Morris S. Ultrasonography as a diagnostic tool in assessing deltoid ligament injury in supination external rotation fractures of the ankle. Orthopedics. 2011;34(10):639-643.

16.Lantto I, Heikkinen J, Flinkkila T, Ohtonen P, Kangas J, Siira P, Leppilahti J. Early Functional Treatment Versus Cast Immobilization in Tension After Achilles Rupture Repair: Results of a Prospective Randomized Trial With 10 or More Years of Follow-up. The American Journal of Sports Medicine. 2015;43(9):2302-2309.

17.Leppilahti J, Forsman K, Puranen J, Orava S. Outcome and prognostic factors of Achilles rupture repair using a new scoring method. Clinical orthopaedics and related research. 1998;346:152-161.

18.Maffulli N, Tallon C, Wong J, Lim KP, Bleakney R. Early weightbearing and ankle mobilization after open repair of acute midsubstance tears of the achilles tendon. Am J Sports Med. 2003;31(5):692-700.

19.Maffulli N, Tallon C, Wong J, Lim KP, Bleakney R. Open Repair Of Acute Midsubstance Tears Of The Achilles Tendon: Early Weightbearing And Ankle Mobilisation. Orthopaedic Proceedings. 2005;87(3):375-380.

20.McCormack R, Bovard J. Early functional rehabilitation or cast immobilisation for the postoperative management of acute Achilles tendon rupture? A meta-analysis of randomised controlled trials. Br J Sports Med. 2015;49(20):1329-1335.

21.Nilsson-Helander K, Silbernagel KG, Thomee R, Faxen E, Olsson N, Eriksson BI, Karlsson J. Acute Achilles tendon rupture: a randomized, controlled study comparing surgical and nonsurgical treatments using validated outcome measures. The American journal of sports medicine. 2010;38(11):2186-2193.

22.Olsson N. Acute Achilles Tendon Rupture. Outcome, Prediction and Optimized treatment. Gothenburg: Sweden; 2013.

23.Olsson N, Silbernagel KG, Eriksson BI, Sansone M, Brorsson A, Nilsson-Helander K, Karlsson J. Stable Surgical Repair With Accelerated Rehabilitation Versus Nonsurgical Treatment for Acute Achilles Tendon Ruptures. The American journal of sports medicine. 2013;41(12):2867-2876.

24.Pettengill KM. The Evolution of Early Mobilization of 
the Repaired Flexor Tendon. Journal of Hand Therapy. 2005;18(2):157-168.

25.Qin L, Appell H-J, Chan KM, Maffulli N. Electrical stimulation prevents immobilization atrophy in skeletal muscle of rabbits. Arch. Phys. and Med. Rehab. 1997;78:512517.

26.Thermann H. Management of Achilles tendon rupture. Orthopade. 1999;28(1):82-97.
27.Willits K, Amendola A, Bryant D, Mohtadi NG, Giffin JR, Fowler P, Kean CO, Kirkley A. Operative versus nonoperative treatment of acute Achilles tendon ruptures: a multicenter randomized trial using accelerated functional rehabilitation. J Bone Joint Surg Am. 2010;92(17):2767-2775.

\section{Information about the authors:}

Vitomskyi V.V.; http://orcid.org/0000-0002-4582-6004; vitomskiyvova@rambler.ru; SU «Scientific-Practical Medical Center of Pediatric Cardiology and Cardiac Surgery of the MH of Ukraine», 24 Melnikov St, Kyiv, 04050, Ukraine; National University of Physical Education and Sport of Ukraine; 1 Phizkultury Street, Kiev, 03680, Ukraine.

Lazarieva O.B.; http://orcid.org/0000-0002-7435-2127; helenka__@mail.ru; National University of Physical Education and Sport of Ukraine; 1 Phizkultury Street, Kiev, 03680, Ukraine.

Ra'ad Abdul Hadi Mohammad Alalwan; http://orcid.org/0000-0003-1268-0894; raad_olwan@yahoo.com; National University of Physical Education and Sport of Ukraine; The University of Jordan; 1 Phizkultury Street, Kiev, 03680, Ukraine.

Vitomska M.V.; http://orcid.org/0000-0002-5163-3954; vitomskiyvova@rambler.ru; National University of Physical Education and Sport of Ukraine; 1 Phizkultury Street, Kiev, 03680, Ukraine.

Cite this article as: Vitomskyi VV, Lazarieva OB, Ra'ad Abdul Hadi Mohammad Alalwan, Vitomska MV. Restoration of ankle joint, quality of life dynamics and assessment of achilles tendon rupture consequences. Pedagogics, psychology, medical-biological problems of physical training and sports, 2017;21(6):308-314. doi:10.15561/18189172.2017.0608

The electronic version of this article is the complete one and can be found online at: http://www.sportpedagogy.org.ua/index.php/PPS/issue/archive

This is an Open Access article distributed under the terms of the Creative Commons Attribution License, which permits unrestricted use, distribution, and reproduction in any medium, provided the original work is properly cited (http://creativecommons.org/licenses/by/4.0/deed.en).

Received: 19.05.2017

Accepted: 15.06.2017; Published: 05.11.2017 